\title{
On the Knowledge-based Dynamic Fuzzy Sets
}

\author{
Rolly Intan, Siana Halim, Lily Puspa Dewi \\ Petra Christian University, Surabaya 60236, Indonesia \\ rintanepetra.ac.id
}

\begin{abstract}
In 1965, L.A. Zadeh [1] introduced fuzzy set as a generalization of crisp set by considering membership degrees of elements. That membership degree is represented gradually as a real number in an interval [0,1]. Wang et al. [2] generalized the concept of fuzzy set, called Dynamic Fuzzy Sets (DFS). In the DFS each membership degree of an element is given by a membership function dealing with time variable. Thus, the membership degree of an element in a given dynamic fuzzy set might dynamically change according to the time's variable. In 2002, Intan and Mukaidono [3] proposed an extended concept of fuzzy set, called Knowledge-based Fuzzy Sets (KFS). In the KFS, the membership degree of an element given a fuzzy set is subjectively determined by a single knowledge. The membership degree of an element with respect to a given fuzzy set may be different provided by different knowledge of persons. This paper combines both concepts, DFS and KFS, called Knowledge-based Dynamic Fuzzy Sets (KDFS), by realizing that membership function of a given fuzzy set provided by a certain knowledge may be dynamically changed over time. Three kinds of summary fuzzy sets are proposed and discussed. Some basic operations of KDFS are defined. Their properties are verified and examined.
\end{abstract}

Keywords: Fuzzy Sets, Dynamic Fuzzy Sets, Knowledge-based Fuzzy Sets, Knowledge-based Dynamic Fuzzy Set

\section{Introduction}

Classical sets, known as a crisp set, are used to represent collections of objects in which every object as a member of a given set is characterized by a membership degree. Since every element is only recognized either as a member or non-member, membership degree of an element is then expressed by a binary number in $\{0,1\}$, where membership degree of 0 means the element is a non-member, and membership degree of 1 means the element is a member.

In the development of sets theory, several generalized concepts of crisp sets were proposed to be applicable in simulating a complex real-world problem. Rough sets theory was introduced by Pawlak in 1982[4] with a practical goal of representing indiscernibility of objects or elements dealing with an information system, a table of objects characterized by a set of attributes. Rough sets are a generalization of crisp sets by introducing a formulation of sets with imprecise boundaries. A rough set is expressed conceptually as an approximation of a given crisp set into two subsets of approximation, 
called lower and upper approximations. Multisets, also known as bags, are also considered as another generalization of crisp sets by allowing multiple occurrences for each of its elements in a multiset Blizard 1991 [5]. The term multiset was first time introduced by Nicolaas Govert de Bruijn in 1970 [6]. However, the concept of multisets has been used since many centuries ago, long before Nicolaas Govert de Bruijn introduced the word multiset. The fuzzy set was introduced by L.A. Zadeh in 1965 [1,7]. By contrast to crisp sets, membership degrees of elements in a fuzzy set is represented gradually by a real number in an interval $(0,1)$. So, the membership degree of an element in a given fuzzy set could be gradually started from 0 (non-member) to 1 (member) [8]. Thus, a fuzzy set is also determined as a generalization of a crisp set, i.e., a crisp set is only a special case of fuzzy set.

Membership degree of a given fuzzy set is determined and calculated by a membership function without any consideration to the time variable. In other words, ones when a membership degree of an element is determined by a membership function, dealing with a change of time, it will stay unchangeably. However, it is obviously known that everything in the world is always changing in connection with the change of time. Membership degree of an element given a fuzzy set is possibly changeable with respect to the time variable. Therefore, Wang et al. [2] proposed a concept of set, called $D y$ namic Fuzzy Sets (DFS). The DFS is an extended concept of fuzzy sets, i.e., each membership degree of an element in DFS is given by a membership function with time's variable. Here, membership degree of an element in a given dynamic fuzzy set might dynamically change according to time's variable. The DFS may be considered as fuzzy multisets dealing with time variable. Practically, the concept of dynamic fuzzy sets can be used to represent or to simulate the change of anything related to the change of time as usually happened in the real world application. The concept of DFS [2], and discussed by Cai et al. [9] as part of his proposed concept, called shadow fuzzy sets, is different from the concept of DFS discussed by Zhang [10]. In this case, the concept of DFS discussed by Zhang [10] was an extension of intuitionistic fuzzy sets proposed by K. T. Atanassov [11].

Considering the membership function of a given fuzzy set is subjectively determined by certain knowledge, Intan and Mukaidono $[2,12,13]$ proposed and discussed a generalized concept of fuzzy sets, called Knowledge-based Fuzzy Sets (KFS). Here, fuzziness may be regarded as deterministic uncertainty. It means that even in uncertain (unclear) situation or definition of an object, a subject (person) through his/ her knowledge may be subjectively able to determine the object. Thus, a given fuzzy set may have $n$ different membership functions related to $n$ different knowledge. Similarly, the knowledgebased fuzzy sets may be also regarded as other example of fuzzy multisets dealing with knowledge.

It has possibly happened, a membership function of a fuzzy set given by certain knowledge is changeable over time. Thus, it is necessary to propose a new concept, called Knowledge-based Dynamic Fuzzy Sets (KDFS) as a hybrid concept of DFS and KFS. Here the KDFS may also be considered as a concept of two-dimensional fuzzy multisets. Three kinds of summary fuzzy sets are proposed and discussed. They are the knowledge-based summary fuzzy sets, the time-based summary fuzzy sets and the gen- 
eral summary fuzzy sets. Some basic operations of KDFS such as equality, contentment, union, intersection and complement are defined. Their properties are verified and examined.

\section{Dynamic Fuzzy Sets and Knowledge-based Fuzzy Sets}

Dynamic Fuzzy Sets and Knowledge-based Fuzzy sets may be considered as more practical example of fuzzy multisets as proposed by Yager [14] and Miyamoto [15,16]. Thus, they may be also regarded as the generalization of fuzzy sets in a more practical use. The concepts of dynamic fuzzy sets and knowledge-based fuzzy sets are briefly discussed as follows.

\subsection{Dynamic Fuzzy Sets}

Considering that membership function of a given fuzzy set is possibly changeable dealing with time variable, Wang et al. [2] introduced an extended concept of fuzzy sets, called Dynamic Fuzzy Sets as the following definition.

Definition 1 Let $U$ be a universal set of elements and $T \subseteq R^{+}$be a set of time, where $R^{+}=[0, \infty)$. Then a dynamic fuzzy set $A$ on $U$ is defined and characterized by the following membership function.

$$
A: T \times U \rightarrow[0,1]
$$

$A(t, u) \in[0,1]$ is the membership degree of element $u$ to dynamic fuzzy set $A$ at the time $t \in T$. Obviously, $A(t, u)=1$ means $u$ is a full member of $A$ at the time $t \in T$. On the other hand, $A(t, u)=0$ means $u$ is not a member of $A$. Thus, the membership degree of an element $u$ may vary depending on the time $t \in T . A(t) \in \mathcal{F}(U)$ is regarded as a dynamic fuzzy set $A$ at the time $t \in T$ which is a similar concept to the fuzzy set defined by Zadeh in 1965 [1], where $\mathcal{F}(U)$ is a fuzzy power set of $U$. Set of dynamic fuzzy sets $A$ on $U$, denoted by $\mathcal{T}(A)$ is given by: $\mathcal{T}(A)=\{A(t) \mid t \in T\}$.

\subsection{Knowledge-based Fuzzy Sets}

As discussed by Intan and Mukaidono [3,12,13], the uncertainty might be categorized into two types, deterministic uncertainty and non-deterministic uncertainty. Fuzziness may be regarded as a deterministic uncertainty. It means that even in uncertain (unclear) situation or definition of an object, a subject (person) through his/ her knowledge is able to determine the object subjectively. Similarly, as what happened in fuzziness, someone may subjectively determine the membership function of a given fuzzy set using his/ her knowledge. Different knowledge may have different membership function of a given fuzzy set. Thus, $n$-knowledge may have $n$ different membership function of a given fuzzy set. Here, knowledge plays significant roles in determining membership 
function of a given fuzzy set. A concept of knowledge-based fuzzy set is defined as follows.

Definition 2 Let $U$ be a universal set of elements, and $K$ be a set of knowledges. Then a knowledge-based fuzzy set $A$ on $U$ based on the knowledge $k \in K$, denoted by $A_{k}$ is defined and characterized by the following membership function.

$$
A_{k}: U \rightarrow[0,1]
$$

Similarly, $A_{k}(u) \in[0,1]$ is the membership degree of element $u \in U$ to fuzzy set $A$ based on knowledge $k \in K$. Obviously, $A_{k}(u)=1$ means $u$ is a full member of $A$ based on $k$. On the other hand, $A_{k}(u)=0$ means $u \in U$ is not a member of $A_{k}$. Thus, the membership degree of an element $u$ to $A$ may vary depending on knowledge $k \in K$. $A_{k} \in \mathcal{F}(U)$ is regarded as a knowledge-based fuzzy set of $A$ based on knowledge $k$ which is also a similar concept to the fuzzy set defined by Zadeh in 1965 [1,7], where $\mathcal{F}(U)$ is a fuzzy power set of $U$. Set of knowledge-based fuzzy sets $A$ on $U$, denoted by $\mathcal{K}(A)$ is given by: $\mathcal{K}(A)=\left\{A_{k} \mid k \in K\right\}$.

\section{Knowledge-based Dynamic Fuzzy Sets}

As the primary objective of this paper, we introduce a new concept, called Knowledgebased Dynamic Fuzzy Sets as a hybrid concept between dynamic fuzzy sets and knowledge-based fuzzy sets. Practically in the real world application, even a certain membership function of fuzzy set $A$ has already given by a certain knowledge $k$, the membership function is possibly changeable over the time. The following definition starts the concept of knowledge-based dynamic fuzzy sets.

Definition 3 Let $U$ be a universal set of elements, $K$ be a set of knowledge and $T \subseteq$ $R^{+}$be a set of time, where $R^{+}=[0, \infty)$. Then a knowledge-based dynamic fuzzy set $A$ on $U$ based on the knowledge $k \in K$, denoted by $\delta_{k}^{A}$, is defined and characterized by the following membership function.

$$
\delta_{k}^{A}: T \times U \rightarrow[0,1]
$$

Related to (3), $\delta_{k}^{A}(t, u) \in[0,1]$ is the membership degree of element $u \in U$ to fuzzy set $A$ based on knowledge $k \in K$ at the time $t \in T$. Similarly, $\delta_{k}^{A}(t, u)=1$ means $u$ is a full member of $A$ based on $k$ at the time $t$. On the other hand, $\delta_{k}^{A}(t, u)=0$ means $u \in U$ is not a member of $\delta_{k}^{A}(t)$. Thus, the membership degree of an element $u$ to $A$ may also vary depending on both the knowledge $k$ and the time $t$. Here, $\delta_{k}^{A}(t) \in \mathcal{F}(U)$ is regarded as a knowledge-based dynamic fuzzy set of $A$ which is based on knowledge $k$ at the time $t . \delta_{k}^{A}(t)$ is also a similar concept to the fuzzy set defined by Zadeh $[1,6]$, where $\mathcal{F}(U)$ is a fuzzy power set of $U$. Set of knowledge-based dynamic fuzzy sets $A$ on $U$, denoted by $\mathcal{D}(A)$ is given by: $\mathcal{D}(A)=\left\{\delta_{k}^{A}(t) \mid k \in K, t \in T\right\}$. 


\subsection{Summary Fuzzy Sets}

The relation among dynamic fuzzy sets (DFS), knowledge-based fuzzy sets (KFS) and knowledge-based dynamic fuzzy sets (KDFS) may be represented by Table 1 . Let $A$ be a fuzzy set on $U, K$ be a set of knowledge and $T$ be a set of times, where $K=$ $\left\{k_{1}, k_{2}, \ldots, k_{n}\right\}$ and $T=\left\{t_{1}, t_{2}, \ldots, t_{m}\right\}$.

Table 1. Relation among DFS, KFS and KDFS

\begin{tabular}{|c|c|c|c|}
\hline & $A\left(t_{1}\right)$ & $\cdots$ & $A\left(t_{m}\right)$ \\
\hline$A_{k_{1}}$ & $\delta_{k_{1}}^{A}\left(t_{1}\right)$ & $\cdots$ & $\delta_{k_{1}}^{A}\left(t_{m}\right)$ \\
\hline$\vdots$ & $\vdots$ & $\ddots$ & $\vdots$ \\
\hline$A_{k_{n}}$ & $\delta_{k_{n}}^{A}\left(t_{1}\right)$ & $\cdots$ & $\delta_{k_{n}}^{A}\left(t_{m}\right)$ \\
\hline
\end{tabular}

For every $t_{j} \in T$ and $k_{i} \in K$, it can be furtherly interpreted that both $A_{k_{i}}$ and $A\left(t_{j}\right)$ are able to be provided by two aggregation functions over $\delta_{k_{i}}^{A}\left(t_{j}\right)$ as follows.

$$
\begin{aligned}
& \forall u \in U, A_{k_{i}}(u)=\Upsilon\left(\delta_{k_{i}}^{A}\left(t_{1}, u\right), \cdots, \delta_{k_{i}}^{A}\left(t_{m}, u\right)\right), \text { where } \Upsilon:[0,1]^{m} \rightarrow[0,1] \\
& \forall u \in U, A\left(t_{j}, u\right)=\Theta\left(\delta_{k_{1}}^{A}\left(t_{j}, u\right), \cdots, \delta_{k_{n}}^{A}\left(t_{j}, u\right)\right), \text { where } \Theta:[0,1]^{n} \rightarrow[0,1]
\end{aligned}
$$

Here, depending on the context of applications, $\Upsilon$ and $\Phi$ may use any existed functions such as maximum, minimum, average, etc. by taking the maximum and the minimum functions as the maximum and the minimum values of both functions, respectively. Eq. (5) is exactly similar to the knowledge-based summary fuzzy set as discussed by Intan and Mukaidono [3,12,13]. Practically, the knowledge-based summary fuzzy set of $A$ as formulated in (5) means an agreement given by a group of persons represented by a set of knowledge to describe $A$ at the time $t_{j}$. Similarly, Eq. (4) may be regarded to provide the time-based summary fuzzy set. For it is sometimes happened in reality, subjective opinion of someone to a given fuzzy set $A$ may be changeable according to the changing of times, the objective of (4) is to summarize the multiple opinions of a certain knowledge $k_{i}$ to the fuzzy set $A$. Therefore, related to the reason behind calculating both summary fuzzy sets as given in (4) and (5), it may be more applicable to use the weighted average as the aggregation function as shown in the following equations.

$$
\begin{gathered}
\Upsilon\left(\delta_{k_{i}}^{A}\left(t_{1}, u\right), \cdots, \delta_{k_{i}}^{A}\left(t_{m}, u\right)\right)=\frac{\sum_{j=1}^{m} w_{j} \cdot \delta_{k_{i}}^{A}\left(t_{j}, u\right)}{\sum_{j=1}^{m} w_{j}}, \text { where } w_{j} \in R^{+}, R^{+}=[0, \infty) \\
\Theta\left(\delta_{k_{1}}^{A}\left(t_{j}, u\right), \cdots, \delta_{k_{n}}^{A}\left(t_{j}, u\right)\right)=\frac{\sum_{i=1}^{n} w_{i} \cdot \delta_{k_{i}}^{A}\left(t_{j}, u\right)}{\sum_{i=1}^{n} w_{i}}, \text { where } w_{i} \in R^{+}, R^{+}=[0, \infty)
\end{gathered}
$$

Related to the summary fuzzy sets, $w_{i}$ and $w_{j}$ are weights to express the prominence of an opinion. For instance, in the case of the knowledge-based summary fuzzy sets, more prominent knowledge $k_{i}$ is considered in determining the summary fuzzy set, a larger $w_{i}$ is given to $k_{i}$. In the case of the time-based summary fuzzy sets, a larger weight may 
be given generally to the more recent opinion, since a more current opinion represent a more real-time situation. So, in the case of constructing the time-based summary fuzzy sets, the relation between time and weight should satisfy $t_{j}>t_{p} \Rightarrow w_{j} \geq w_{p}, \forall t_{j}, t_{p} \in$ $T$.

It is also necessary to summarize all interpretation/ opinion based on the knowledge as well as the times into only one summary fuzzy set, called general summary fuzzy set. Here, the general summary fuzzy sets may be assumed as an agreement, to sum up all opinions given by multiple pieces of knowledge over the time. Given $A$ be a fuzzy set on $U$. Let $K=\left\{k_{1}, \cdots, k_{n}\right\}$ and $T=\left\{t_{1}, \cdots, t_{m}\right\}$. Using the concept of weighted average, we introduce three different equations to provide the general summary fuzzy set as follows.

- Aggregated from the Knowledge-based Summary Fuzzy Sets

$$
A^{G_{1}}(u)=\Psi\left(A_{k_{1}}(u), \cdots, A_{k_{n}}(u)\right)=\frac{\sum_{i=1}^{n} w_{i} \cdot A_{k_{i}}(u)}{\sum_{i=1}^{n} w_{i}},
$$

where $w_{i} \in R^{+}, R^{+}=[0, \infty)$.

- Aggregated from the Time-based Summary Fuzzy Sets

$$
A^{G_{2}}(u)=\Gamma\left(A\left(t_{1}, u\right), \cdots, A\left(t_{m}, u\right)\right)=\frac{\sum_{j=1}^{m} w_{j} \cdot A\left(t_{j}, u\right)}{\sum_{j=1}^{m} w_{j}},
$$

where $w_{i} \in R^{+}, R^{+}=[0, \infty)$

- Aggregated directly from the Knowledge-based Dynamic Fuzzy Sets

$$
A^{G_{3}}(u)=\Omega\left(\begin{array}{ccc}
\delta_{k_{1}}^{A}\left(t_{1}, u\right) & \cdots & \delta_{k_{1}}^{A}\left(t_{m}, u\right) \\
\vdots & \ddots & \vdots \\
\delta_{k_{n}}^{A}\left(t_{1}, u\right) & \cdots & \delta_{k_{n}}^{A}\left(t_{m}, u\right)
\end{array}\right)=\frac{\sum_{j=1}^{n} \sum_{i=1}^{m} w_{i j} \cdot \delta_{k_{i}}^{A}\left(t_{j}, u\right)}{\sum_{j=1}^{n} \sum_{i=1}^{m} w_{i j}},
$$

where $w_{i j} \in R^{+}, R^{+}=[0, \infty)$.

These three different equations may produce different results that it depends on the context of application to decide which one is better to use.

\subsection{Basic Operations and Properties}

Some basic operations of the knowledge-based dynamic fuzzy sets may be discussed and defined as follows.

Definition 4 Let $U$ be a universal set of elements, $K$ be a set of knowledges and $T \subseteq$ $R^{+}$be a set of time, where $R^{+}=[0, \infty) . A$ and $B$ are two fuzzy sets on $U$. Some basic operations and properties of Equality, Containment, Union, Intersection and Complementation are given by the following equations.

Equality 
1. $\delta_{k}^{A}(t)=\delta_{k}^{B}(t) \Leftrightarrow \delta_{k}^{A}(t, u)=\delta_{k}^{B}(t, u), \forall u \in U$,

2. $A_{k}=B_{k} \Leftrightarrow A_{k}(u)=B_{k}(u), \forall u \in U$,

3. $A_{k} \equiv B_{k} \Leftrightarrow \delta_{k}^{A}(t, u)=\delta_{k}^{B}(t, u), \forall u \in U, \forall t \in T$,

4. Similarly, $A_{k} \equiv B_{k} \Leftrightarrow \delta_{k}^{A}(t)=\delta_{k}^{B}(t), \forall t \in T$,

5. $A(t)=B(t) \Leftrightarrow A(t, u)=B(t, u), \forall u \in U$,

6. $A(t) \equiv B(t) \Leftrightarrow \delta_{k}^{A}(t, u)=\delta_{k}^{B}(t, u), \forall u \in U, \forall k \in K$,

7. Similarly, $A(t) \equiv B(t) \Leftrightarrow \delta_{k}^{A}(t)=\delta_{k}^{B}(t), \forall k \in K$,

8. $A=B \Leftrightarrow \delta_{k}^{A}(t, u)=\delta_{k}^{B}(t, u), \forall u \in U, \forall k \in K, \forall t \in T$,

9. $A \cong B \Leftrightarrow \delta_{k}^{A}\left(t_{j_{1}}, u\right)=\delta_{k}^{B}\left(t_{j_{2}}, u\right), \forall u \in U, \forall k \in K, \forall t_{j_{1}}, t_{j_{2}} \in T$,

10. $A \triangleq B \Leftrightarrow \delta_{k_{i_{1}}}^{A}(t, u)=\delta_{k_{i_{2}}}^{B}(t, u), \forall u \in U, \forall k_{i_{1}}, k_{i_{2}} \in K, \forall t \in T$,

11. $A \equiv B \Leftrightarrow \delta_{k_{i_{1}}}^{A}\left(t_{j_{1}}, u\right)=\delta_{k_{i_{2}}}^{B}\left(t_{j_{2}}, u\right), \forall u \in U, \forall k_{i_{1}}, k_{i_{2}} \in K, \forall t_{j_{1}}, t_{j_{2}} \in T$,

12. $k_{i_{1}}=k_{i_{2}} \Leftrightarrow \delta_{k_{i_{1}}}^{A}(t, u)=\delta_{k_{i_{2}}}^{A}(t, u), \forall u \in U, \forall t \in T, A \in \mathcal{F}(U)$, where $\mathcal{F}(U)$ is fuzzy power set on $U$.

\section{Containment}

13. $\delta_{k}^{A}(t) \subseteq \delta_{k}^{B}(t) \Leftrightarrow \delta_{k}^{A}(t, u) \leq \delta_{k}^{B}(t, u), \forall u \in U$,

14. $A_{k} \subseteq B_{k} \Leftrightarrow A_{k}(u) \leq B_{k}(u), \forall u \in U$,

15. $A_{k} \Subset B_{k} \Leftrightarrow \delta_{k}^{A}(t, u) \leq \delta_{k}^{B}(t, u), \forall u \in U, \forall t \in T$,

16. Similarly, $A_{k} \subseteq B_{k} \Leftrightarrow \delta_{k}^{A}(t) \subseteq \delta_{k}^{B}(t), \forall t \in T$,

17. $A(t) \subseteq B(t) \Leftrightarrow A(t, u) \leq B(t, u), \forall u \in U$,

18. $A(t) \Subset B(t) \Leftrightarrow \delta_{k}^{A}(t, u) \leq \delta_{k}^{B}(t, u), \forall u \in U, \forall k \in K$,

19. Similarly, $A(t) \subseteq B(t) \Leftrightarrow \delta_{k}^{A}(t) \leq \delta_{k}^{B}(t), \forall k \in K$,

20. $A \subseteq B \Leftrightarrow \delta_{k}^{A}(t, u) \leq \delta_{k}^{B}(t, u), \forall u \in U, \forall k \in K, \forall t \in T$,

21. $A \subseteq B \Leftrightarrow \delta_{k}^{A}\left(t_{j_{1}}, u\right) \leq \delta_{k}^{B}\left(t_{j_{2}}, u\right), \forall u \in U, \forall k \in K, \forall t_{j_{1}}, t_{j_{2}} \in T$,

22. $A \preccurlyeq B \Leftrightarrow \delta_{k_{i_{1}}}^{A}(t, u) \leq \delta_{k_{i_{2}}}^{B}(t, u), \forall u \in U, \forall k_{i_{1}}, k_{i_{2}} \in K, \forall t \in T$,

23. $A \subseteq B \Leftrightarrow \delta_{k_{i_{1}}}^{A}\left(t_{j_{1}}, u\right) \leq \delta_{k_{i_{2}}}^{B}\left(t_{j_{2}}, u\right), \forall u \in U, \forall k_{i_{1}}, k_{i_{2}} \in K, \forall t_{j_{1}}, t_{j_{2}} \in T$,

24. $k_{i_{1}} \unlhd k_{i_{2}} \Leftrightarrow \delta_{k_{i_{1}}}^{A}(t, u) \leq \delta_{k_{i_{2}}}^{A}(t, u), \forall u \in U, \forall t \in T, A \in \mathcal{F}(U)$, where $\mathcal{F}(U)$ is fuzzy power set on $U$.

\section{Union}

25. $\delta_{k}^{A \cup B}(t, u)=\max \left(\delta_{k}^{A}(t, u), \delta_{k}^{B}(t, u)\right), \forall u \in U$,

26. $\left(\delta_{k_{i_{1}}}^{A}\left(t_{j_{1}}\right) \cup \delta_{k_{i_{2}}}^{B}\left(t_{j_{2}}\right)\right)(u)=\max \left(\delta_{k_{i_{1}}}^{A}\left(t_{j_{1}}, u\right), \delta_{k_{i_{2}}}^{B}\left(t_{j_{2}}, u\right)\right), \forall u \in U, t_{j_{1}}, t_{j_{2}} \in T$, $k_{i_{1}}, k_{i_{2}} \in K$,

27. $\left(A_{k} \cup B_{k}\right)(u)=(A \cup B)_{k}(u)=\max \left(A_{k}(u), B_{k}(u)\right), \forall u \in U$,

28. $\left(A_{k_{i_{1}}} \cup B_{k_{i_{2}}}\right)(u)=\max \left(A_{k_{i_{1}}}(u), B_{k_{i_{2}}}(u)\right), \forall u \in U, k_{i_{1}}, k_{i_{2}} \in K$,

29. $(A(t) \cup B(t))(u)=(A \cup B)(t, u)=\max (A(t, u), B(t, u)), \forall u \in U$,

30. $\left(A\left(t_{j_{1}}\right) \cup B\left(t_{j_{2}}\right)\right)(u)=\max \left(A\left(t_{j_{1}}, u\right), B\left(t_{j_{2}}, u\right)\right), \forall u \in U, t_{j_{1}}, t_{j_{2}} \in T$,

31. $(A \cup B)(u)=\max \left(A^{G_{p}}(u), B^{G_{p}}(u)\right), \forall u \in U, p=\{1,2,3\}$, where $A^{G_{p}}$ and $B^{G_{p}}$ are general fuzzy sets as given in (8), (9) and (10). 


\section{Intersection}

32. $\delta_{k}^{A \cap B}(t, u)=\min \left(\delta_{k}^{A}(t, u), \delta_{k}^{B}(t, u)\right), \forall u \in U$,

33. $\left(\delta_{k_{i_{1}}}^{A}\left(t_{j_{1}}\right) \cap \delta_{k_{i_{2}}}^{B}\left(t_{j_{2}}\right)\right)(u)=\min \left(\delta_{k_{i_{1}}}^{A}\left(t_{j_{1}}, u\right), \delta_{k_{i_{2}}}^{B}\left(t_{j_{2}}, u\right)\right), \forall u \in U, t_{j_{1}}, t_{j_{2}} \in T$, $k_{i_{1}}, k_{i_{2}} \in K$,

34. $\left(A_{k} \cap B_{k}\right)(u)=(A \cap B)_{k}(u)=\min \left(A_{k}(u), B_{k}(u)\right), \forall u \in U$,

35. $\left(A_{k_{i_{1}}} \cap B_{k_{i_{2}}}\right)(u)=\min \left(A_{k_{i_{1}}}(u), B_{k_{i_{2}}}(u)\right), \forall u \in U, k_{i_{1}}, k_{i_{2}} \in K$,

36. $(A(t) \cap B(t))(u)=(A \cap B)(t, u)=\min (A(t, u), B(t, u)), \forall u \in U$,

37. $\left(A\left(t_{j_{1}}\right) \cap B\left(t_{j_{2}}\right)\right)(u)=\min \left(A\left(t_{j_{1}}, u\right), B\left(t_{j_{2}}, u\right)\right), \forall u \in U, t_{j_{1}}, t_{j_{2}} \in T$,

38. $(A \cap B)(u)=\min \left(A^{G_{p}}(u), B^{G} p(u)\right), \forall u \in U, p \in\{1,2,3\}$, where $A^{G_{p}}$ and $B^{G_{p}}$ are general fuzzy sets as given in (8), (9) and (10).

\section{Complementation}

39. $\delta_{k_{i}}^{\neg A}\left(t_{j}, u\right)=1-\delta_{k_{i}}^{A}\left(t_{j}, u\right)$,

40. $\delta_{\neg k_{i}}^{A}\left(t_{j}, u\right)=\left\{\begin{array}{c}\delta_{k_{r}}^{A}\left(t_{j}, u\right), r \neq i,|K|=2, \\ \Theta\left(\alpha_{k_{1}}, \cdots, \alpha_{k_{i-1}}, \alpha_{k_{i+1}}, \cdots, \alpha_{k_{n}}\right),|K|>2, \alpha_{k_{p}}=\delta_{k_{p}}^{A}\left(t_{j}, u\right),\end{array}\right.$

41. $\delta_{k_{i}}^{A}\left(\neg t_{j}, u\right)=\left\{\begin{array}{c}\delta_{k_{i}}^{A}\left(t_{r}, u\right), r \neq i,|T|=2, \\ \Upsilon\left(\beta_{t_{1}}, \cdots, \beta_{t_{j-1}}, \beta_{t_{j+1}}, \cdots, \beta_{t_{m}}\right),|T|>2, \beta_{t_{p}}=\delta_{k_{i}}^{A}\left(t_{p}, u\right),\end{array}\right.$

42. $\neg A_{k_{i}}(u)=1-A_{k_{i}}(u)$,

43. $A_{\neg k_{i}}(u)=\left\{\begin{array}{c}A_{k_{r}}(u), r \neq i,|K|=2, \\ \Psi\left(A_{k_{1}}(u), \cdots, A_{k_{i-1}}(u), A_{k_{i+1}}(u), \cdots, A_{k_{n}}(u)\right),|K|>2,\end{array}\right.$

44. $\neg A\left(t_{j}, u\right)=1-A\left(t_{j}, u\right)$,

45. $A\left(\neg t_{j}, u\right)=\left\{\begin{array}{c}A\left(t_{r}, u\right), r \neq j,|K|=2, \\ \Gamma\left(A\left(t_{1}, u\right), \cdots, A\left(t_{j-1}, u\right), A\left(t_{j+1}, u\right), \cdots, A\left(t_{m}, u\right)\right),|K|>2,\end{array}\right.$

46. $\neg A(u)=1-A^{G_{p}}, p \in\{1,2,3\}$, where $A^{G_{p}}$ and $B^{G_{p}}$ are general fuzzy sets as given in (8), (9) and (10).

There are some properties as a consequence of the basic operations as given in Definition 4 as follows.

- From Equality 2, 3 and 4: $A_{k} \equiv B_{k} \Rightarrow A_{k}=B_{k}, \forall k \in K$.

- From Equality 5, 6 and 7: $A(t) \equiv B(t) \Rightarrow A(t)=B(t), \forall t \in T$.

- From Equality 8 to $11:(A \equiv B) \Rightarrow\{(A \cong B),(A \triangleq B)\} \Rightarrow(A=B)$.

- From Containment 14, 15 and 16: $A_{k} \Subset B_{k} \Rightarrow A_{k} \subseteq B_{k}, \forall k \in K$.

- From Containment 17, 18 and 19: $A(t) \Subset B(t) \Rightarrow A(t) \subseteq B(t), \forall t \in T$.

- From Containment 20 to 23: $A \Subset B) \Rightarrow\{(A \subseteq B),(A \preccurlyeq B)\} \Rightarrow(A \subseteq B)$. 


\section{Conclusion}

In the real-world application, it is possible that a membership function of a fuzzy set subjectively given by a certain knowledge is changeable over time. Therefore, this paper introduced a generalized concept of fuzzy sets, called knowledge-based dynamic fuzzy sets. The concept is a hybrid concept of dynamic fuzzy sets proposed by Wang et al. [2] and knowledge-based fuzzy sets introduced and discussed by Intan and Mukaidono $[3,10,11]$. The concept of knowledge-based dynamic fuzzy sets is regarded as a practical example of two-dimensional fuzzy multisets dealing with time and knowledge variables. Three kinds of summary fuzzy sets, namely the knowledge-based summary fuzzy sets, the time-based summary fuzzy sets and the general summary fuzzy sets are proposed and discussed using aggregation functions. Some basic operations such as equality, containment, union, intersection and complementation are defined. Their properties are verified and examined.

\section{References}

1. Zadeh, L.A.: Fuzzy Sets. Inf. Control 8, 338-353, (1965).

2. Wang, G.Y., Ou, J.P., Wang, P.Z.: Dynamic fuzzy sets. Fuzzy System Math 2(1), 1-8, (1988).

3. Intan, R., Mukaidono, M.: On Knowledge-based Fuzzy Sets. International Journal of Fuzzy Systems 4(2), Chinese Fuzzy Systems Association T (CFSAT), 655-664, (2002).

4. Pawlak, Z.: Rough Sets. International Journal Computation and Information Science 11, 341-356. (1982).

5. Blizard, Wayne D.: The Development of Multiset Theory. Modern Logic 1(4), 319-352, (1991).

6. de Bruijn, N. G.: A Generalization of Burnside's lemma, Technical Report, Notitie 55, Technological University Eindhoven (1970).

7. Zadeh, L.A.: Fuzzy Sets and systems. International Journal of General Systems 17, 129-138, (1990).

8. Klir, G.J., Yuan, B.: Fuzzy Sets and Fuzzy Logic: Theory and Applications, New Jersey: Prentice Hall, (1995).

9. Cai, M., Li, Q., Lang, G.: Shadowed sets of dynamic fuzzy sets. Granular Computing 2, 8594, (2017).

10. Zhang, Z.: A Novel Dynamic Fuzzy Sets Method Applied to Practical Teaching Assessment on Statistical Software. IERI Procedia 2, 303-310, (2012).

11. Atanassov, K.T.: Intuitionistic Fuzzy Sets. Fuzzy Sets and System 20, 87-96, (1986).

12. Intan, R., Mukaidono, M., Emoto, M.: Knowledge- based Representation of Fuzzy Sets. Proceeding of FUZZ-IEEE'02, 590-595, (2002).

13. Intan, R., Mukaidono, M.: Approximate Reasoning in Knowledge-based Fuzzy Sets. Proceeding of NAFIPS-FLINT 2002, IEEE Publisher, 439-444, (2002).

14. Yager, R.R.: On the Theory of Bags. International Journal of General Systems 13, 23-37, (1986).

15. Miyamoto, S.: Fuzzy Multisets and their Generalizations. Multiset Processing 2235, 225235, (2001).

16. Miyamoto, S.: Fuzzy Sets in Information Retrieval and Cluster Analysis. Kluwer Academic Publishers, (1990). 